\title{
Caris Molecular Intelligence Tumor Profiling
}

National Cancer Institute

\section{Source}

National Cancer Institute. Caris Molecular Intelligence Tumor Profiling. NCI Thesaurus.

Code C153292.

A proprietary genomic profiling that assess DNA, RNA and proteins to establish a

molecular profile for use in guiding individualized treatment decisions. 